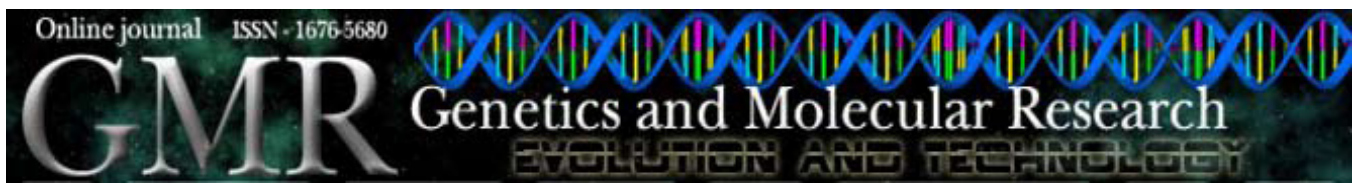

\title{
Programmed cell death in salivary glands of Drosophila arizonae and Drosophila mulleri
}

\author{
P. Ianella, M.T.V. Azeredo-Oliveira and M.M. Itoyama \\ Laboratório de Citogenética de Insetos, Departamento de Biologia, \\ Universidade do Estado de São Paulo, IBILCE, São José do Rio Preto, SP, Brasil \\ Corresponding author: M.M. Itoyama \\ E-mail: mary@ibilce.unesp.br
}

Genet. Mol. Res. 7 (2): 476-486 (2008)

Received January 20, 2008

Accepted May 2, 2008

Published June 3, 2008

\begin{abstract}
Programmed cell death (PCD) in insect metamorphosis assumes a great diversity of morphology and controlling processes that are still not well understood. With the objective of obtaining information about the PCD process, salivary glands of Drosophila arizonae and $D$. mulleri were studied during larval-pupal development. From the results, it can be concluded that the type of the PCD that occurs in these organs is morphologically typical of apoptosis (formation of apoptotic nuclei, followed by fragmentation into apoptotic bodies). Histolysis happens in both species, between 22 and $23 \mathrm{~h}$ after pupation. There were no significant differences between the species studied. Apoptosis does not occur simultaneously in all cells. Cytoplasmic acid phosphatase activity gradually increases during development, suggesting the existence of acid phosphatases that are only expressed during the apoptotic stage. Twenty hours after pupation, salivary glands already show biochemical alterations relative to nuclear permeability such as acidification, possibly due to the fusion of lysosomes with the nucleus a few hours before apoptosis. Autophagy seems to act together with apoptosis and has a secondary role in cell death.
\end{abstract}

Key words: Drosophila; Programmed cell death; Salivary gland; Development; Acid phosphatase; Acridine orange 


\section{INTRODUCTION}

Cells initiating their own destruction is a common phenomenon in both invertebrate and vertebrate development (Bowen and Lockshin, 1981). Selective cell death is seen as a fundamental process during the embryogenesis (Saunders Jr., 1966) of insects and amphibian morphogenesis, which involves massive and coordinated cell destruction of the transient tissues (Bowen and Lockshin, 1981). Metamorphosis of holometabolous insects carries a radical reconstruction of the body planes to a replacement of the larval tissues by adult tissues and structures. Processes that drive these changes are controlled by the insect steroid molting hormone ecdysone (Jiang et al., 1997, 2000; Sekimoto et al., 2006).

In Drosophila, almost all organs are destroyed and new structures develop in their places from embryonic cell groups that go on undifferentiated in the larval period (Robertson, 1936). It is known that the histolysis of these tissues occurs through programmed cell death (PCD). However, there are many controversies regarding the type of PCD that occurs in insect metamorphosis.

PCD is characteristic of normal tissues and it can show several distinct morphological and biochemical patterns. Classic apoptosis and autophagy are the two most prominent morphological forms of cell death that occur during animal developmental processes (Schweickel and Merker, 1973; Clarke, 1990). The former is marked by cell contraction, nuclear shrinkage (forming pycnotic nuclei) with subsequent fragmentation into apoptotic bodies, and endonuclease activation. The latter process, less known, implies the formation of autophagic vacuoles that are used for cytoplasmic destruction.

Jiang et al. (1997) have shown that in Drosophila melanogaster salivary gland histolysis is triggered by steroid hormones. Many researchers have described apparently nonapoptotic or aberrant apoptotic modes of PCD during insect development (Schwartz, 1991) and metamorphosis (Lockshin and Zakeri, 1990; Bowen et al., 1993; Silva de Moraes and Bowen, 2000). According to Bowen et al. (1993), who studied cell death in the salivary glands of Calliphora vomitoria during development, the classical apoptotic marker (DNA fragmentation and degeneration) does not apply to this model. Nevertheless, Gregorc and Bowen (1997) and Silva de Moraes and Bowen (2000), studying respectively salivary glands and midgut of the Apis mellifera larva, observed DNA fragmentation frequently associated with cell death. Dai and Gilbert (1997) concluded that both modes of PCD occur in the salivary glands of Manduca sexta. The results of Armbruster et al. (1986) and Levy and Bautz (1985), in Calliphora vomitoria, allow us to infer that both modes of PCD (apoptosis and autophagy) coexist in these species' salivary glands. The PCD of Drosophila salivary glands has been considered to be autophagic by Lee and Baehrecke (2001), Baehrecke (2003) and Yin and Thummel (2005); however, these authors did not discard the possibility that these mechanisms act together. Martin and Baehrecke (2004) concluded that studies of salivary glands indicate that the distinction between autophagic cell death and apoptosis seems more subtle than the morphology suggests.

Apoptotic cells can be easily visualized in Drosophila embryos with a vital stain, such as acridine orange. The mode by which this stain acts in apoptotic cells is not known, although many mechanisms are possible, including an increase in nuclear membrane permeability. In living cells, acridine orange serves as a $\mathrm{pH}$ indicator, becoming trapped in acid compartments such as lysosomes (Zelenin, 1966).

It is known that during tissue histolysis of holometabolous insects, some enzymatic functions, such as acid phosphatase activity, undergo changes as a consequence 
of morphological and functional cell collapse. Acid phosphatases consist of a group of nonspecific enzymes that hydrolyze a variety of organic esters with the release of phosphate ions and the optimum $\mathrm{pH}$ for these enzymes varies from 4.5 to 6.0 (Essner, 1973). They have been considered to be lysosomal markers, but extralysosomal sites have been detected. Many researchers (Bowen et al., 1993; Gregorc and Bowen, 1997) have associated their activities with the eminence of cell death, and therefore, acid phosphatases have been studied during insect metamorphosis. This enzymatic group acts by tissue histolytic degradation. Bowen and Lockshin (1981) observed that the loss of larval tissues occurring during Drosophila metamorphosis is accompanied by an increase in both histochemical frequency and biochemical activity of lysosomes. Jones and Bowen (1993) described both the biochemical profile and the structural localization of acid phosphatase activity in Drosophila larval salivary glands and observed that before histolysis there is an increase in this enzymatic activity related to PCD. The same pattern was observed in several other species of insects (Aidells et al., 1971; Schin and Laufer, 1973; Armbruster et al., 1986; Bowen, 1993; Bowen et al., 1996; Gregorc and Bowen, 1997).

In view of such unconformity in the literature related to the mode of PCD occurring in holometabolous insects and the characteristics involved in the process, the main goal of the current paper was to identify the kind of cell death occurring in the salivary glands of $D$. arizonae and D. mulleri. We also aimed to establish at which moment of the pupal-adult transition the histolysis of this tissue PCD occurs, and whether there are significant differences between the two species studied, and to establish a relationship between acid phosphatase activities and PCD in these organisms.

\section{MATERIAL AND METHODS}

The sympatric species $D$. arizonae and D. mulleri (repleta group) were maintained in cactus culture medium at a constant temperature of $20 \pm 1^{\circ} \mathrm{C}$, in a $1 / 4$ liter bottle. Late thirdinstar larvae (3L), pre-pupae (PP; larvae with spiracle everted, but with no rigid covering), pupae (pre-pupae aged) of 8 (P8), 16 (P16), 20 (P20), 22 (P22), 22:30 (P22:30), and 23 h (P23) were used to analyze salivary gland cells. Salivary glands were extracted from individuals in the stages mentioned above and submitted either to the Feulgen reaction (except P20) or acid phosphatase reaction (modified from Gomori, 1950). A third technique, staining with acridine orange, was used like a vital stain (Abrams et al., 1993).

Feulgen reaction. Glands were submitted to acid hydrolysis using $4 \mathrm{~N} \mathrm{HCl}$ for $65 \mathrm{~min}$ at room temperature. After that, a fast rinse in $0.1 \mathrm{~N} \mathrm{HCl}$ was done to block the hydrolysis. Afterwards, the material was stained with Schiff's reagent for $40 \mathrm{~min}$ at $37^{\circ} \mathrm{C}$ and submitted to three successive rinses ( 5 min each) in sulfur water, and finally rinsed three times in distilled water.

Acid phosphatase reaction. Unfixed salivary glands were incubated for $45 \mathrm{~min}$ in phosphate buffer, $\beta$-glycerophosphate, and lead nitrate, at $37^{\circ} \mathrm{C}$, and later rinsed in phosphate buffer. Afterwards, they were treated with $1 \%$ ammonium sulfate for $2 \mathrm{~min}$, rinsed in distilled water and placed in glycerin.

Acridine orange. The glands were submitted to $5 \mu \mathrm{g} / \mathrm{mL}$ acridine orange solution $(0.1$ $\mathrm{M}$ phosphate buffer, $\mathrm{pH}$ 7.2) for $5 \mathrm{~min}$. The slides were analyzed and the images were captured by an AXIOSCOP 2 ZEISS light microscope. For fluorescent analysis, a green filter (FITC) was used. 


\section{RESULTS}

In the present study, the salivary glands of 3L, $\mathrm{PP}$ and pupae of $D$. arizonae and $D$. mulleri were analyzed by staining with the Feulgen reaction, acid phosphatase reaction and acridine orange technique. The salivary glands from $3 \mathrm{~L}$ to $\mathrm{P} 16$ (Figures $1 \mathrm{~A}, \mathrm{~B}$ and $2 \mathrm{~A}, \mathrm{~B}$ ) of the two species analyzed did not show nuclear morphological alterations related to PCD events. P22, of both species, displayed histolysis signs (Figures 1C,D and 2C,D), such as pycnotic nuclei, which are typical of apoptotic cells. D. mulleri salivary glands at P22:30 were found in a more advanced stage of degeneration. At this stage, we observed typical nuclei of this tissue, pre-apoptotic and apoptotic nuclei, and apoptotic bodies resulting from the fragmentation of apoptotic nuclei (Figures 1E,F and 2E,F). In the glands of P23 of both species, we could see more advanced stages of histolysis, since only pre-apoptotic (pycnotic nuclei) and apoptotic nuclei were found. A great number of these apoptotic nuclei were disintegrating into apoptotic bodies (Figures 1G,H and 2G,H).

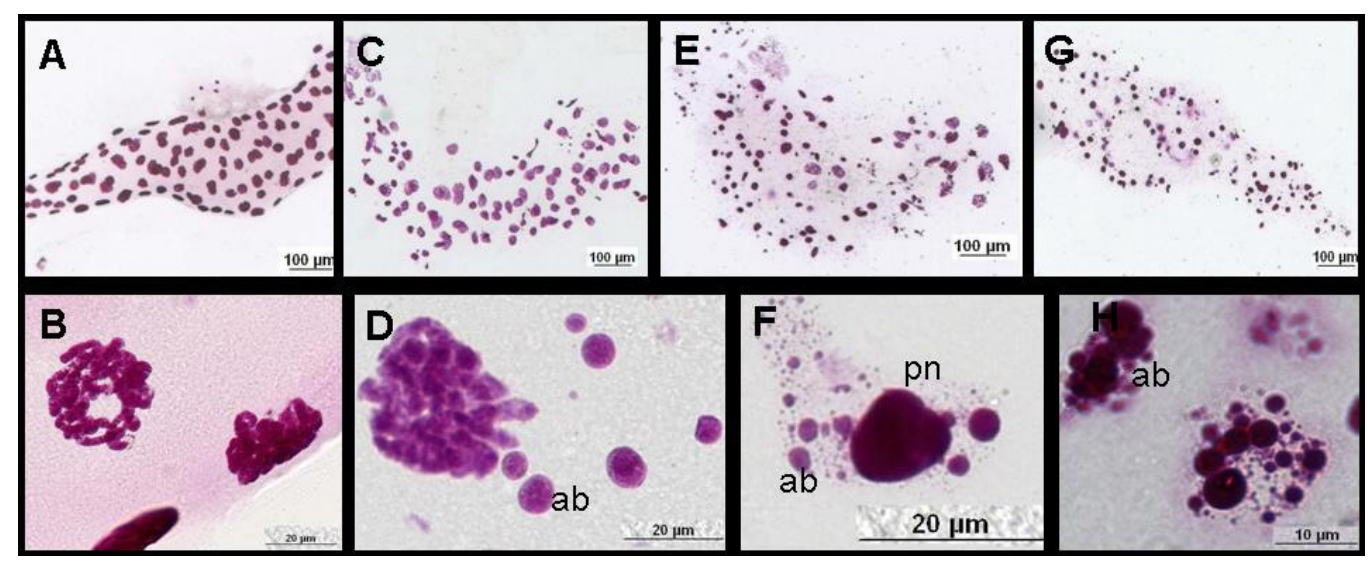

Figure 1 A-H. Salivary gland cells of Drosophila arizonae. A,B: P16; C,D: P22; E,F: P22:30; G,H: P23. ab = apoptotic bodies; $p n=$ pre-apoptotic nuclei.

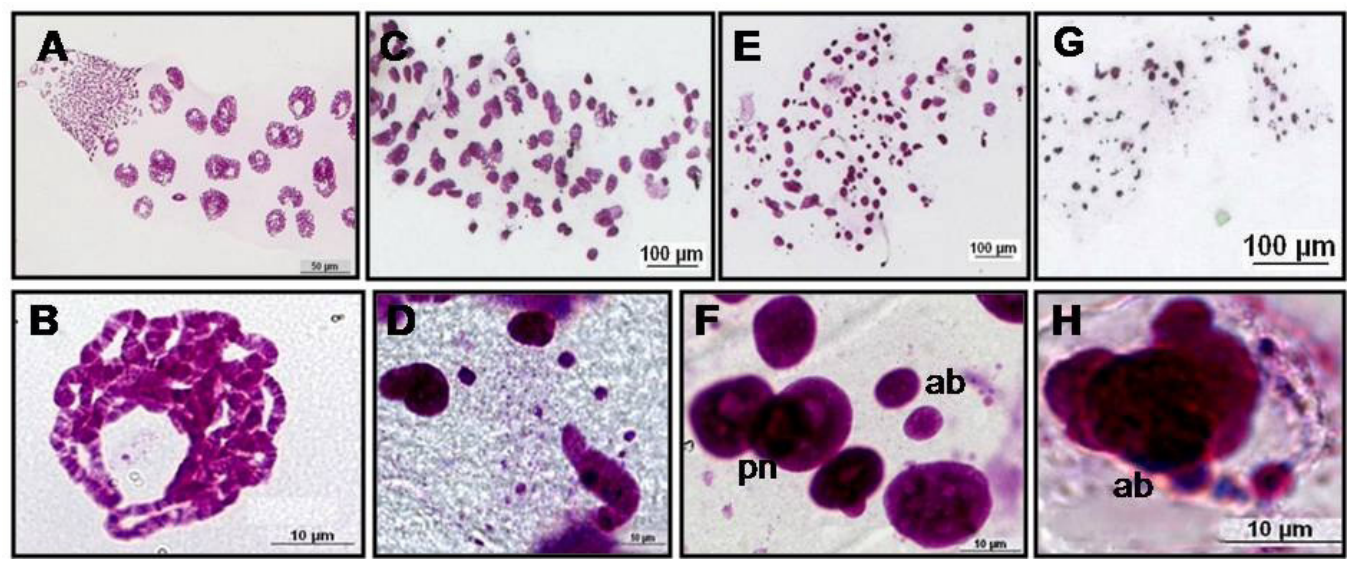

Figure 2 A-H. Salivary gland cells of Drosophila mulleri. A,B: P16; C,D: P22; E,F: P22:30; G,H: P23. ab = apoptotic bodies; $\mathrm{pn}=$ pre-apoptotic nuclei. 
Whole glands of all developmental stages submitted to acid phosphatase reaction were positively stained by this technique. In the salivary glands of third-instar larvae (3T) of D. arizonae (Figure 3A,B) and D. mulleri (Figure 4A,B), acid phosphatase staining occurred in the cytoplasmic, nucleolar and nuclear (specifically on the chromosomes) regions. In glands of PP (Figures 3C,D and 4C,D) and P8 (Figures 3E,F and 4E,F) of both species, the nucleolus was strongly stained (Figures 3E,F and 4E,F), but the nucleus on the whole was less stained than in $3 \mathrm{~T}$ glands. The glands of this stage showed a gradual increase in cytoplasmic staining.
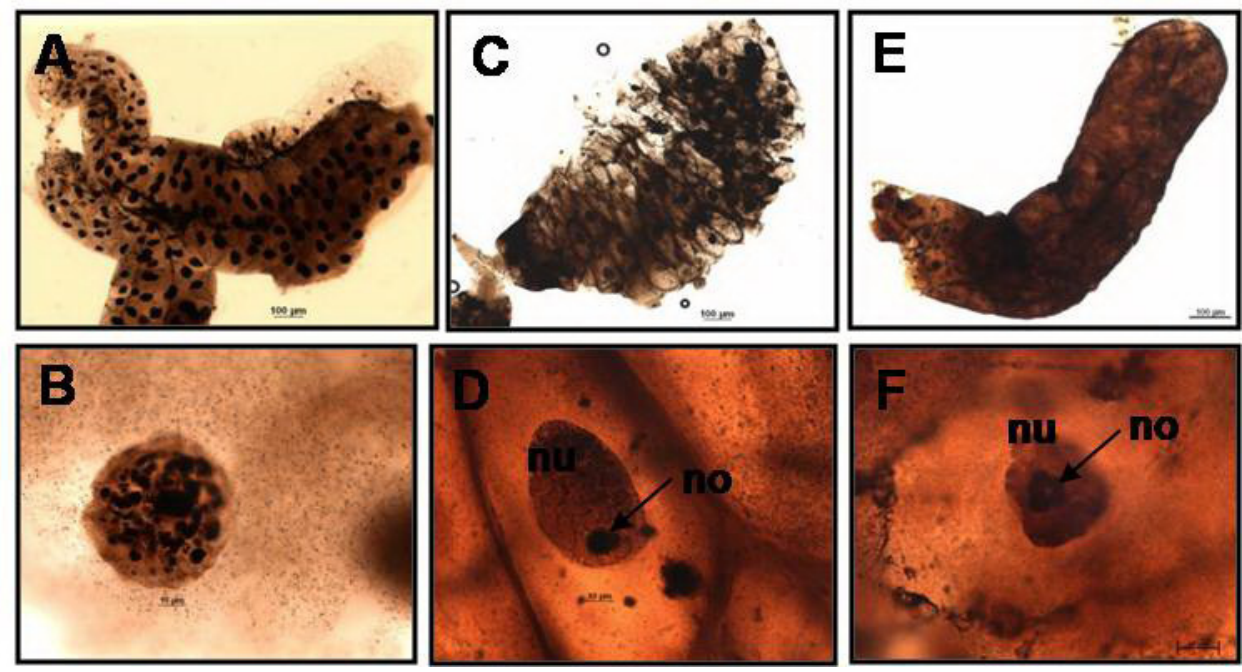

Figure 3 A-F. Salivary gland cells of Drosophila arizonae. A,B: 3T; C,D: PP; E,F: P8. nu = nucleus; no = nucleolus.
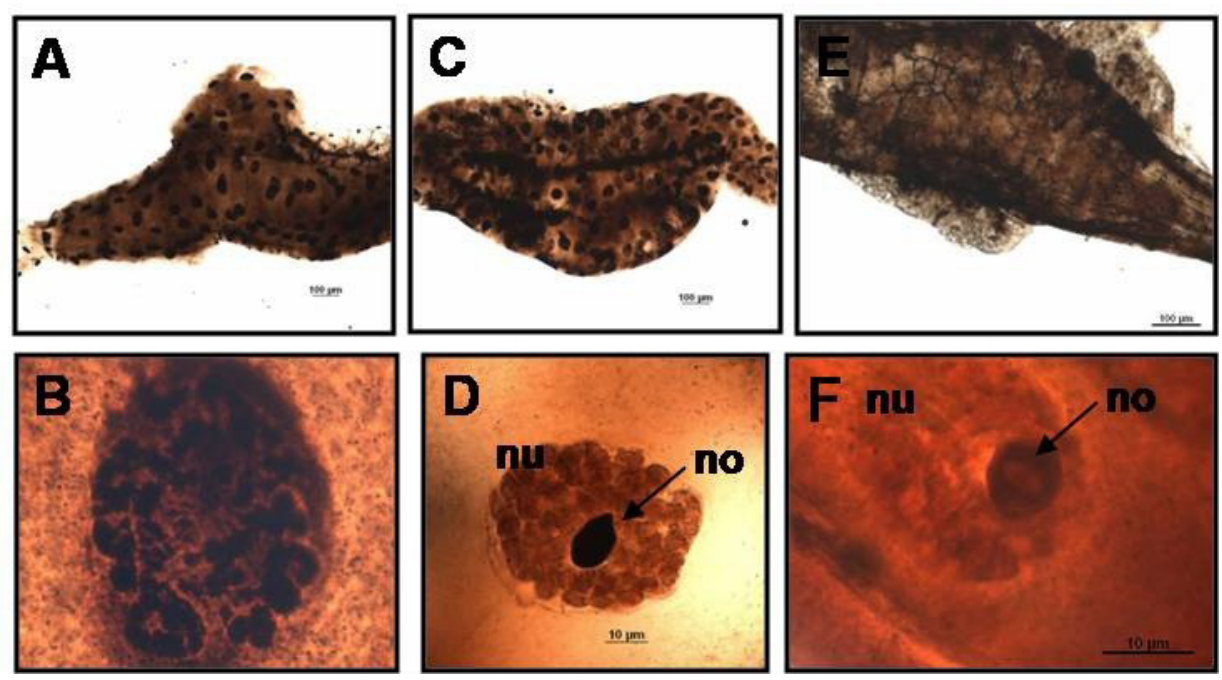

Figure 4 A-F. Salivary gland cells of Drosophila mulleri. A,B: 3T; C,D: PP; E,F: P8. nu = nucleus; no = nucleolus. 
In D. arizonae (Figure 5A,B) and D. mulleri (Figure 6A,B) pupal glands P16, strong cytoplasmic staining in whole gland was observed, and in the nucleus, the polytene chromosomes were intensively stained. P22, P22:30 and P23 glands of both species studied (Figures $5 \mathrm{C}-\mathrm{H}$ and $6 \mathrm{C}-\mathrm{H}$ ) showed the cytoplasm and nucleus completely stained. Preapoptotic nuclei in D. arizonae (Figure 5D) and D. mulleri (Figure 6D), apoptotic nuclei (Figure 6D) and apoptotic bodies in D. arizonae (Figure 5F and H) and D. mulleri (Figure $6 \mathrm{~F}$ and $\mathrm{H})$ were completely stained.

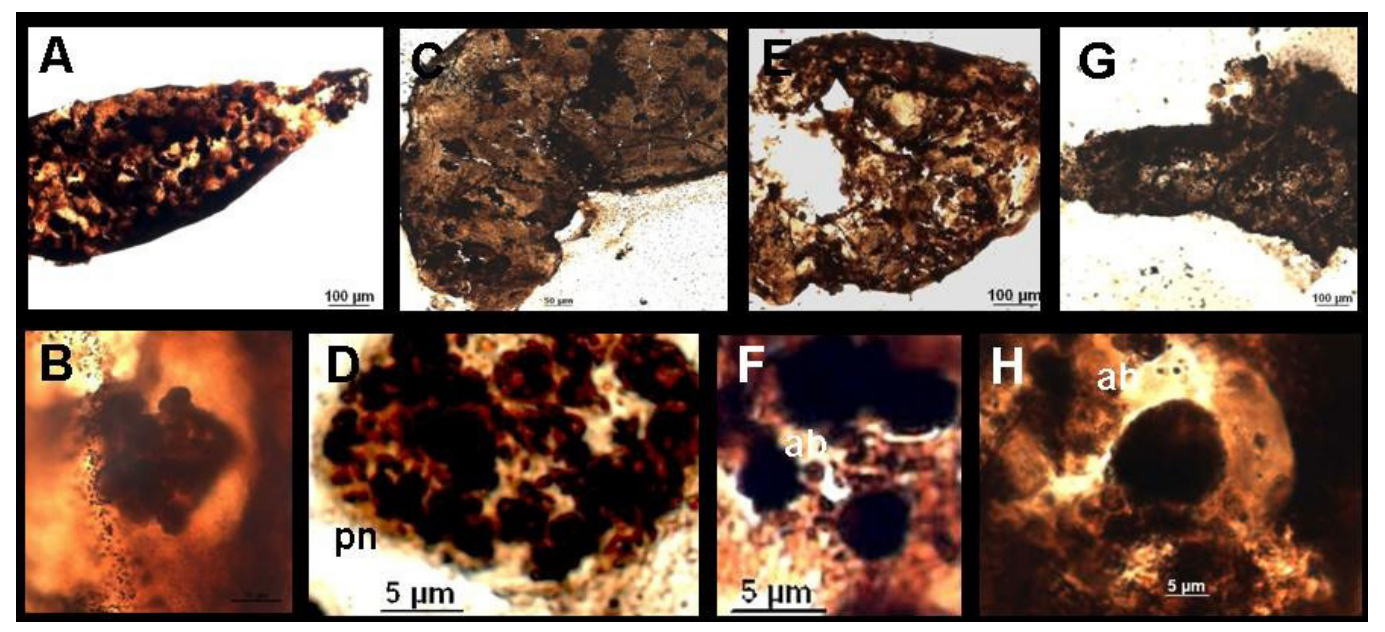

Figure 5 A-H. Salivary gland cells of Drosophila arizonae. A,B: P16; C,D: P22; E,F: P22:30; G,H: P23. ab = apoptotic bodies; pn = pre-apoptotic nuclei.

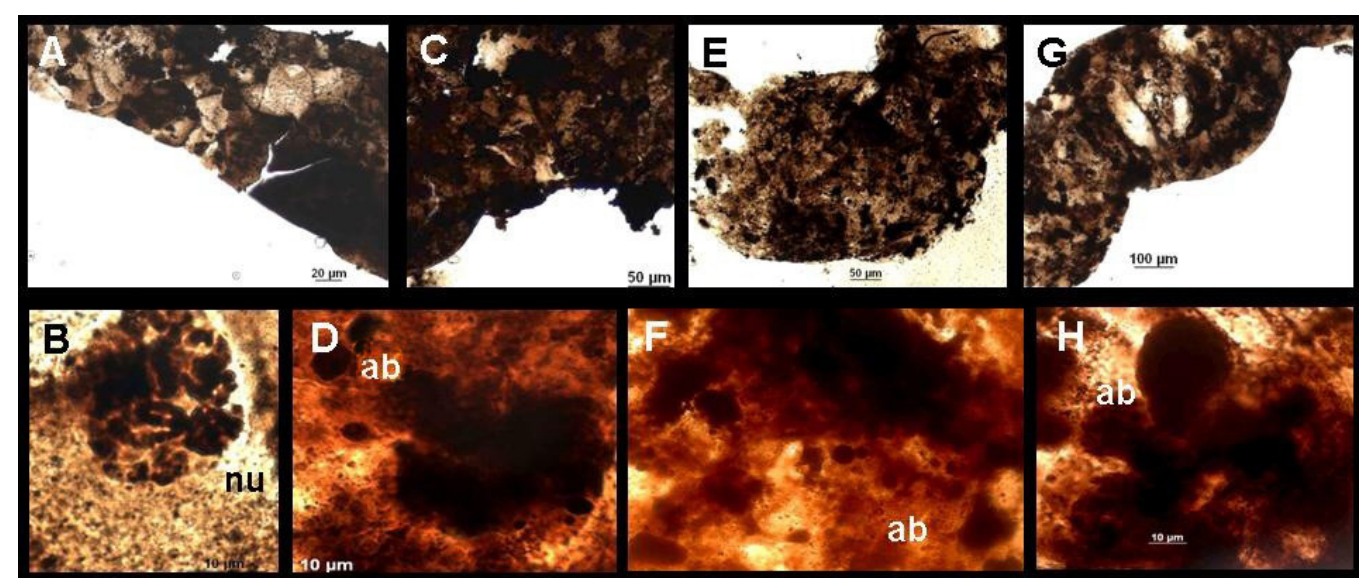

Figure 6 A-H. Salivary gland cells of Drosophila mulleri. A,B: P16; C,D: P22; E,F: P22:30; G,H: P23. nu = nucleus; $a b=$ apoptotic bodies. 
In $3 \mathrm{~T}$ glands of both species stained with acridine orange (Figures 7A,B and 8A,B), a thin coloration in the cell membrane regions could be observed. Cell nuclei in this stage were not stained. The developmental stages PP, P8 and P16 (Figures 7C,D and 8C,D) showed negatively stained nuclei. In D. arizonae (7E,F) and D. mulleri (8E,F) P20, some nuclei were positively stained by acridine orange, meaning that the nucleus was permeable to acridine. The majority of P22 glands of both species (Figures 7G,H and 8G,H) showed nuclei that were positively stained.

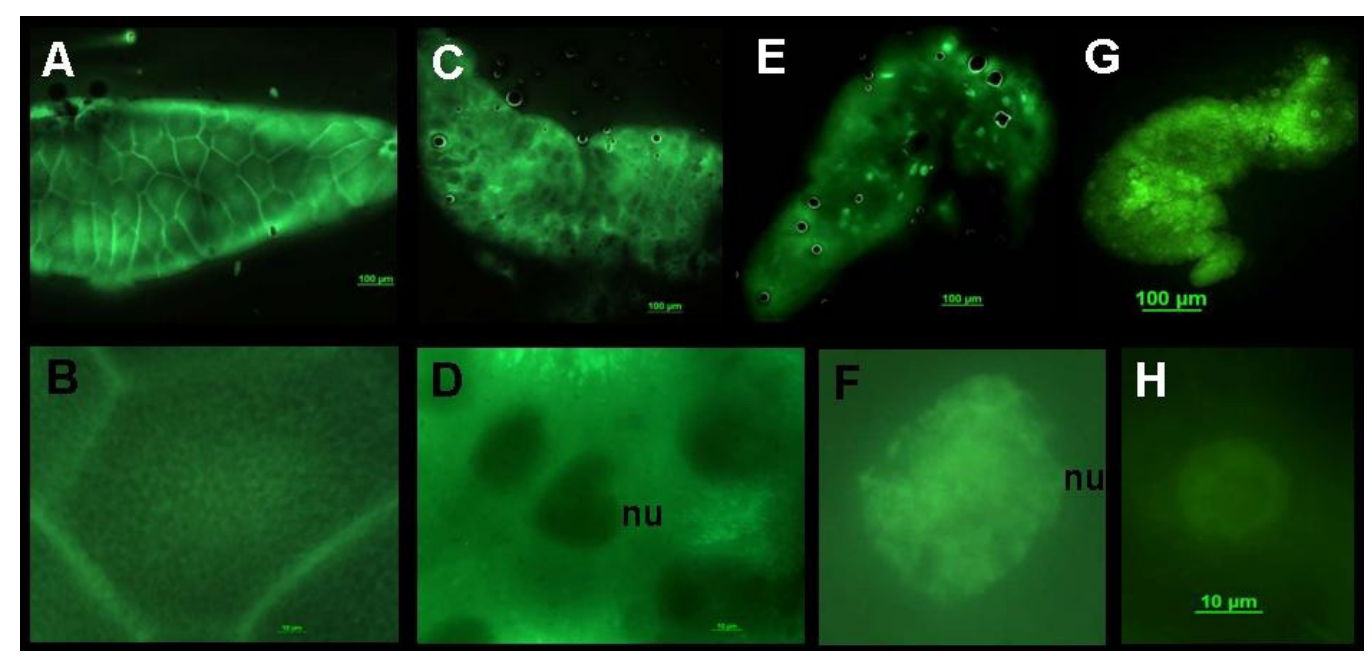

Figure 7 A-H. Salivary gland cells of Drosophila arizonae. A,B: 3T; C,D: P16; E,F: P20; G,H: P22. nu = nucleus.

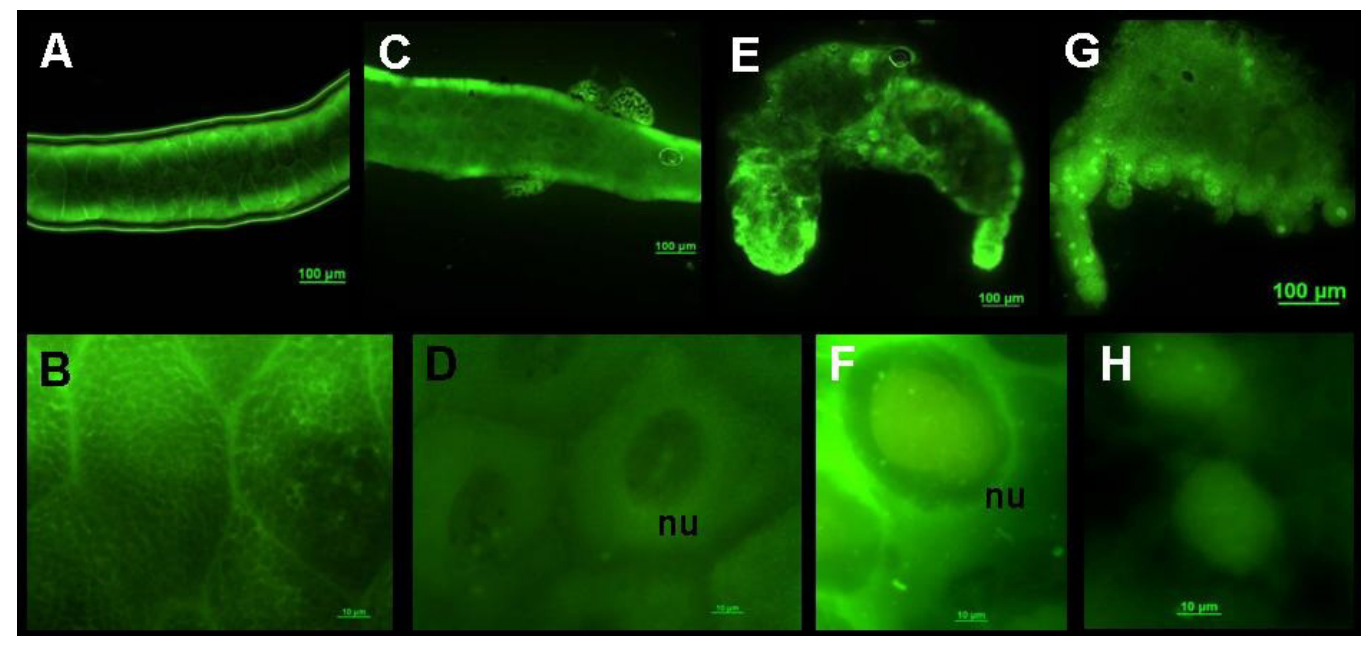

Figure 8 A-H. Salivary gland cells of Drosophila mulleri. A,B: 3T; C,D: P16; E,F: P20; G,H: P22. nu = nucleus. 


\section{DISCUSSION}

The histolysis of Drosophila's salivary glands and of other tissues of holometabolous insects has received considerable attention in the scientific literature. The type of cell death that occurs during histolysis of this organ has been the subject of controversy among researchers. Several of them have assigned this degeneration to the apoptotic mechanism (Berendes and Ashburner, 1978; Jiang et al., 1997; Farkas and Mechler, 2000; Yin et al., 2007), while some to autophagy (Lee and Baehrecke, 2001; Baehrecke, 2003; Yin and Thummel, 2005). Finally, others came to a consensus that the degeneration is a combination of both mechanisms (Levy and Bautz, 1985; Dai and Gilbert, 1997).

In studies by several different authors carried out in holometabolous insects, an excellent model in developmental PCD studies, a large variety of the PCD phenotypes were observed. Bowen et al. (1993), in their studies with Calliphora vomitoria, did not find classic apoptotic traits such as cell shrinkage and chromatin compaction. Lockshin and Zakeri (1990) argued for a separate category of cell death that occurs in insect development, developmental PCD, which specifically does not demonstrate endonuclease activation. These authors have observed that the glands are sculpted by a caspase-mediated PCD in response to an ecdysone pulse, and that the caspase activation begins in the anterior cells and then propagates to the posterior cells. Lee and Baehrecke (2001) and Yin and Thumel (2005) concluded that the PCD activated by hormones occurs by autophagy and that apoptosis and autophagy are distinct processes but use some common regulatory mechanisms. In a study about the rearrangement of the tubulin and actin cytoskeleton during PCD in Drosophila salivary glands, Jochová et al. (1997) obtained results supporting the hypothesis that an extensive autophagic activity occurs during nuclear degradation. Caspase activity has been detected in salivary glands of Drosophila (Takemoto et al., 2007).

Armbruster et al. (1986) inferred that both modalities, apoptosis and autophagy, coexist in Calliphora erythrocephala salivary glands. In this same species, Levy and Bautz (1985) determined that epidermis and salivary gland undergo degeneration by apoptosis, and that autophagic events occur only in the later stages.

Dai and Gilbert (1997) suggested that apoptosis is the basic mechanism for PCD by the way Manduca sexta prothoracic glands undergo histolysis. This hypothesis is supported by Jiang et al. (1997), who studied histolysis of several Drosophila tissues during metamorphosis, triggered by ecdysone. In this study, the authors found DNA fragmentation, among other typical traits of apoptosis. The same was observed recently in salivary glands of Drosophila (Yin et al., 2007).

In the present study, we could observe that in both species the salivary glands start degenerating after $22 \mathrm{~h}$ following pupation, although there is a variation from individual to individual for the time the histolysis begins. However, in general, it seems that there is no morphological variation related to PCD in the developmental stages studied, probably because this organ is the latest to degenerate. According to Jiang et al. (1997), Drosophila post-embryonic metamorphosis is triggered by the steroid hormone ecdysone. The salivary glands degenerate in response to a second pulse of ecdysone that is released late.

In the present study, we could observe in D. arizonae and D. mulleri that cell death does not occur simultaneously in all salivary gland cells and, that it seems that the region where cellular alterations start is random. Thus, we could note in intermediate histolytic phases, morphologically typical nuclei, pre-apoptotic nuclei (nuclei that are already pycnotic but represent an intermediate stage between typical and apoptotic) and apoptotic nuclei. In glands of ad- 
vanced developmental stages, such as P23, we could observe a great number of apoptotic nuclei disintegrating into apoptotic bodies of different sizes, varying from very small to large.

The time of the total degeneration of $D$. arizonae and $D$. mulleri glands may vary from one to one and a half hour, and its beginning occurs usually after $22 \mathrm{~h}$ after PP. With respect to the mode of the PCD that occurs in Drosophila salivary glands, we can affirm that it is morphologically characteristic of apoptosis. The general aspect of the histolysis in this tissue is similar in both species. Apparently, there is no specific characteristic that allows us to differentiate them.

With regard to acid phosphatase activity, we observed that all stages studied showed positive response to acid phosphatase reaction. Cytoplasm and nuclei of all stages were stained, although cytoplasmic activity gradually increased during the period examined. Nuclear staining was also found in the polytene chromosomes, being more intense in 3T (Figures 3B and 4B) and P16 (5B and 6B) than in PP (3D and 4D) and P8 (3F and 4F). According to da Cruz Landim et al. (2002), the heterogeneity in the intensity and distribution of nuclear deposits can be interpreted as a variation in the nuclear functional state. Deltour et al. (1981) and Van de Wall and Bernier (1976) observed the same pattern. Therefore, a relation between nuclear transcription and acid phosphatase activity can be inferred. Such inference can be reinforced because inhibition of transcription is accompanied by acid phosphatase activity suppression in some plants (Walbodt, 1971). These facts allow us to conclude that the stages 3 T and P16 have greater transcriptional activity than the other stages studied.

The nuclear deposits in stages P22 and P23 (period in which morphological characteristics of death by apoptosis can be observed) can be of two kinds: intranuclear granulated deposits (Figure $5 \mathrm{D}$, pre-apoptotic nuclei) and deposits in the whole nuclei (Figure 5F and $\mathrm{H}, 6 \mathrm{H}$ apoptotic nuclei). These kinds of stainings are probably related to acidification of the nucleus. Lu and Wolfe (2001) associated the acid phosphatase activity with the macronucleus degradation event in Tetrahymena. This organism is a unicellular binuclear ciliate that in a determined phase of its cycle has its macronucleus degraded by an apoptotic-like mechanism. These authors observed that lysosomal bodies surround the nuclei before fusing with the nuclei leading to their degradation. Concomitantly, these nuclei become permeable to acridine (a vital stain specific for apoptosis).

Pasteur and Kastritsis (1971) detected biochemically, by microelectrophoresis, the presence of acid phosphatase in whole-fly homogenate and salivary gland extract in all developmental stages analyzed. Whole-fly homogenates compared with salivary gland extracts revealed two coincident bands. These two bands show a similar development pattern in both extracts. One of them disappears at $12 \mathrm{~h}$ after pupation (spiracle evertion). However, the other one increases in intensity after this period, leading to the conclusion that the latter is related to cell death events.

Therefore, we could infer that there are different kinds of phosphatases acting in the nucleus before and during the stages in which apoptosis occurs, but with different functions.

Further studies are needed in order to understand and identify the mechanisms that regulate cell death in different kinds of organisms, which will result in a better understanding of the differences in these fundamental cellular processes.

\section{ACKNOWLEDGMENTS}

The authors thank CNPq (Conselho Nacional de Desenvolvimento Científico e Tecnológico) for the support. 


\section{REFERENCES}

Abrams JM, White K, Fessler LI and Steller H (1993). Programmed cell death during Drosophila embryogenesis. Development 117: 29-43.

Aidells B, Lockshin RA and Cullin AM (1971). Breakdown of the silk glands of Galleria mellonella - acid phosphatase in involuting glands. J. Insect Physiol.17: 857-869.

Armbruster L, Levy M, Mathieu MN and Bautz AM (1986). Acid phosphatase activity in the hemolymph, hemocytes, fat body and salivary glands during larval and prepupal development in Calliphora erythrocephala (Diptera: Calliphoridae). J. Biochem. Physiol. B84: 349-354.

Baehrecke EH (2003). Autophagic programmed cell death in Drosophila. Cell Death Differ. 10: 940-945.

Berendes HD and Ashburner M (1978). The salivary glands. In: The genetics and biology of Drosophila (Ashburner M and Wright TRF, eds.). Academic Press, London, 453-498.

Bowen ID (1993). Apoptosis or programmed cell death? Cell Biol. Int. 17: 365-380.

Bowen ID and Lockshin RA (1981). Cell Death in Biology and Pathology. Chapman and Hall, New York.

Bowen ID, Morgan SM and Mullarkey K (1993). Cell death in the salivary glands of metamorphosing Calliphora vomitoria. Cell Biol. Int. 17: 13-33.

Bowen ID, Mullarkey K and Morgan SM (1996). Programmed cell death during metamorphosis in the blow-fly Calliphora vomitoria. Microsc. Res. Tech. 34: 202-217.

Clarke PG (1990). Developmental cell death: morphological diversity and multiple mechanisms. Anat. Embryol. 181: 195-213.

da Cruz Landim C, Reginato RD, de Moraes RL and Cavalcante VM (2002). Cell nucleus activity during post-embryonic development of Apis mellifera L. (Hymenoptera: Apidae). Intranuclear acid phosphatase. Genet. Mol. Res. 1: 131-138.

Dai JD and Gilbert LI (1997). Programmed cell death of the prothoracic glands of Manduca sexta during pupal-adult metamorphosis. Insect Biochem. Mol. Biol. 27: 69-78.

Deltour R, Fransolet S and Loppes R (1981). Inorganic phosphate accumulation and phosphatase activity in the nucleus of maize embryo root cells. J. Cell Sci. 47: 77-89.

Essner E (1973). Phosphatases. In: Electron microscopy of enzymes (Principles and Methods) (Hayat MA, ed.). Van Nostrand Reinhold Co., New York, 44-76.

Farkas R and Mechler BM (2000). The timing of Drosophila salivary gland apoptosis displays an 1(2)gl-dose response. Cell Death Differ. 7: 89-101.

Gömori G (1950). An improved histochemical technic for acid phosphatase. Stain Technol. 25: 81-85.

Gregorc A and Bowen ID (1997). Programmed cell death in the honey-bee (Apis mellifera L.) larvae midgut. Cell Biol. Int. 21: 151-158.

Jiang C, Baehrecke EH and Thummel CS (1997). Steroid regulated programmed cell death during Drosophila metamorphosis. Development 124: 4673-4683.

Jiang C, Lamblin AF, Steller H and Thummel CS (2000). A steroid-triggered transcriptional hierarchy controls salivary gland cell death during Drosophila metamorphosis. Mol. Cell 5: 445-455.

Jochova J, Zakeri Z and Lockshin RA (1997). Rearrangement of the tubulin and actin cytoskeleton during programmed cell death in Drosophila salivary glands. Cell Death Differ. 4: 140-149.

Jones HE and Bowen ID (1993). Acid phosphatase activity in the larval salivary glands of developing Drosophila melanogaster. Cell Biol. Int. 17: 305-315.

Lee CY and Baehrecke EH (2001). Steroid regulation of autophagic programmed cell death during development. Development 128: 1443-1455.

Levy M and Bautz AM (1985). Degeneration of larval salivary glands during metamorphosis of the blow-fly Calliphora erythrocephala Meigen (Diptera: Calliphoridae). Int. J. Insect Morphol. Embryol. 14: 281-290.

Lockshin RA and Zakeri ZF (1990). Programmed cell death: new thoughts and relevance to aging. J. Gerontol. 45: B135-B140.

Lu E and Wolfe J (2001). Lysosomal enzymes in the macronucleus of Tetrahymena during its apoptosis-like degradation. Cell Death Differ. 8: 289-297.

Martin DN and Baehrecke EH (2004). Caspases function in autophagic programmed cell death in Drosophila. Development 131: 275-284.

Pasteur N and Kastritsis CD (1971). Developmental studies in Drosophila. I. Acid phosphatases, esterases, and other proteins in organs and whole-fly homogenates during development of D. pseudoobscura. Dev. Biol. 26: 525-536.

Robertson CW (1936). The metamorphosis of Drosophila melanogaster, including an accurately timed account of the 
principal morphological changes. J. Morphol. 59: 351-399.

Saunders JW Jr (1966). Death in embryonic systems. Science 154: 604-612.

Schin K and Laufer H (1973). Studies of programmed salivary gland regression during larval-pupal transformation in Chironomus thummi. I. Acid hydrolase activity. Exp. Cell Res. 82: 335-340.

Schwartz LM (1991). The role of cell death genes during development. Bioessays 13: 389-395.

Schweichel JU and Merker HJ (1973). The morphology of various types of cell death in prenatal tissues. Teratology 7 : 253-266.

Sekimoto T, Iwami M and Sakurai S (2006). Coordinate responses of transcription factors to ecdysone during programmed cell death in the anterior silk gland of the silkworm, Bombyx mori. Insect Mol. Biol. 15: 281-292.

Silva de Moraes RL and Bowen ID (2000). Modes of cell death in the hypopharyngeal gland of the honey bee (Apis mellifera 1). Cell Biol. Int. 24: 737-743.

Takemoto K, Kuranaga E, Tonoki A, Nagai T, et al. (2007). Local initiation of caspase activation in Drosophila salivary gland programmed cell death in vivo. Proc. Natl. Acad. Sci. U. S. A. 104: 13367-13372.

Van de Wall C and Bernier G (1976). Sequence of reactivation of ribonucleic acid synthesis during early germination of the maize embryo. Plant Physiol. 57: 632-639.

Walbodt V (1971). RNA metabolism during embryo development and germination of Phaseolus vulgaris. Develop. Biol. 26: 369-379.

Yin VP and Thummel CS (2005). Mechanisms of steroid-triggered programmed cell death in Drosophila. Cell Develop. Biol. 16: 237-243.

Yin VP, Thummel CS and Bashirullah A (2007). Down-regulation of inhibitor of apoptosis levels provides competence for steroid-triggered cell death. J. Cell Biol. 178: 85-92.

Zelenin AV (1966). Fluorescence microscopy of lysosomes and related structures in living cells. Nature 212: 425-426. 